Arteterapia. Papeles de arteterapia y educación para inclusión social ISSN-e 1988-8309

http://dx.doi.org/10.5209/ARTE.57572

\title{
Pina Bausch. Lo que el cuerpo sabe de la guerra y otros desastres
}

\author{
Raquel Pastor Prada ${ }^{1}$
}

Recibido 21 de julio de 2017/ Aceptado 1 de septiembre de 2017

Resumen. La obra coreográfica de Pina Bausch (Solingen, 1940-Wuppertal, 2009) sustentada sobre sus recuerdos de infancia, nos habla de tragedias aterradoras como la guerra que vivió y de otros dramas cotidianos. Una co-biografía construida colectivamente junto a sus bailarines y bailarinas, capaz de cartografiar las miserias y los temores más universales, de manera no razonada, sino corporeizada. La desesperanza y la angustia subidas a escena en una especie de catarsis acompasada y compartida a partir de la experiencia del cuerpo, que busca la conexión con el mundo, el apoyo en los otros, las otras, para calmar el sufrimiento y curar el trauma. Bausch universaliza el drama humano, porque lo que allí sucede nos vincula a todos y todas.

Palabras clave: Pina Bausch; danza teatro; guerra; trauma; cuerpo.

\section{[en] Pina Bausch. What the body knows from war and other disasters}

Abstract. The choreographic work of Pina Bausch (Solingen, 1940-Wuppertal, 2009) supported by her childhood memories, talks us about terrifying tragedies such the war she lived and other daily dramas. A co-biography built collectively with her dancers, capable of mapping the most universal miseries and fears, in an unreasoned, but embodied way. The hopelessness and the anguish mounted on stage in a sort of rhythmic and shared catharsis from the body experience, seeking connection with the world, and support in others, to calm suffering and cure the trauma. Bausch universalizes the human drama, because what happens there binds us all together.

Keywords: Pina Bausch, dance theater, war, trauma, body.

Sumario. 1. Una observadora silenciosa de la guerra. 2. Cómo dar forma a los recuerdos de infancia. 3. El cuerpo como transmisor de la memoria colectiva del ser humano. 4. Catarsis acompañada, acompasada, compartida. 5. Referencias bibliográficas.

Cómo citar: Pastor Prada, R. (2017). Pina Bausch. Lo que el cuerpo sabe de la guerra y otros desastres, en Arteterapia. Papeles de arteterapia y educación para inclusión social 12, 207-217.

Conservatorio Superior de Danza de Madrid "María de Ávila". Departamento de Pedagogía de la Danza. Departamento de Danza Educativa y Comunitaria

E-mail: raquelpastorprada@gmail.com 


\section{Una observadora silenciosa de la guerra.}

When I look back on my childhood, my youth, my period as a student and my time as a dancer and choreographer - then I see pictures. They are full of sounds, full of aroma. And of course full of people who have been and are part of my life. These picture memories from the past keep coming back and searching for a place. Much of what I experienced as a child takes place again much later on the stage ${ }^{2}$.

Pina Bausch, What moves me

En el bello discurso titulado What moves me, que Pina Bausch escribe en 2007 con motivo del Premio Kyoto de la Fundación Inamori, la coreógrafa alemana ${ }^{3}$ hablaba sobre cómo la guerra es una experiencia que no se olvida. Nacida en 1940 en la ciudad de Solingen (Renania del Norte-Westfalia), sufre durante sus primeros años de vida el horror de la segunda Guerra Mundial. Las sirenas de alarma, las bombas, el terror, la devastación, la alteración de la cotidianeidad, la lucha por la supervivencia, la desaparición de cualquier certeza, la desesperanza existencial que se impregna para siempre. A pesar de la guerra, de las miserias y dificultades que encontraría en aquellos tiempos (Adolphe, 2007, pp. 12-13), Bausch también nos describe pasajes a través de los cuales podemos entrever a una niña feliz. Feliz y curiosa.

Even the restaurant in our hotel was highly interesting for me. My parents had to work a great deal and weren't able to look after me. In the evenings, when I was actually supposed to go to bed, I would hide under the tables and simply stay there. I found what I saw and heard very exciting: friendship, love, and quarrels - simply everything that you can experience in a local restaurant like this. I think this stimulated my imagination a great deal. I have always been a spectator. Talkative, I certainly wasn’t. I was more silent . (Bausch, 2007, párr.10)

Podemos imaginar a la pequeña Pina escondida bajo las mesas del café, escuchando, observando, absorbiendo la vida, las vidas. Sin intervenir, silente. Ella es tan pequeña que no entiende el significado de las palabras que escucha, pero sí entiende el mensaje no verbal que encierran los cuerpos. Y la guerra también se vislumbra a través de ellos y sus gestos, expresiones, a través de los movimientos involuntarios que se deslizan, que impregnan la atmósfera de miedo e incertidumbre. La pequeña

2 Cuando rememoro mi infancia, mi juventud, mi período de estudiante y mis años como bailarina y coreógrafa, entonces veo imágenes. Están llenas de sonidos, llenas de aroma. Y por supuesto llenas de gente que ha sido y es parte de mi vida. Estos recuerdos del pasado siguen volviendo y buscando un lugar. Mucho de lo que experimenté cuando era niña toma lugar de nuevo mucho más tarde en el escenario. [trad. propia]

3 El trabajo de Pina Bausch (Solingen, 27 de julio de 1940-Wuppertal, 30 de junio de 2009) abrió caminos intransitados hasta entonces en el campo de la danza-teatro. Franqueó en muchas ocasiones los límites de lo conocido, y los desbordó, los desgarró. Ha sido la inspiración y el motor creativo para muchos que vinieron después. Cambió para siempre el rumbo de la danza contemporánea del último cuarto del siglo pasado.

$4 \quad$ Incluso el restaurante en nuestro hotel era muy interesante para mí. Mis padres tenían que trabajar muchísimo y no podían cuidarme. Por las noches, cuando se suponía que me iba a la cama, me escondía bajo las mesas y simplemente permanecía allí. Encontraba muy emocionante aquello que veía y escuchaba: amistad, amor, y peleas -sencillamente todo aquello que puedes experimentar en un restaurante local como este. Creo que aquello estimuló mucho mi imaginación. Siempre he sido una espectadora. Habladora, ciertamente no era. Era más bien callada. [trad. propia] 
Pina recoge los dramas de la guerra, su cuerpo menudo registra el sufrimiento de las tragedias personales que presencia. Es una observadora silenciosa de la guerra. Una espectadora sigilosa y voraz. Fretard (1995), en su artículo Pina Bausch, l'exorciste, publicado en Le Monde, así lo imaginaba:

L'enfant n'a pas cinq ans qu'elle a déjà créé son aire de jeu entre les tables. Peutêtre attrape-t-elle au vol des conversations, des mots qui terrifient. Les mots de la guerre, de la haine. Elle entend probablement, au loin, les avions de chasse anglais, puis plus tard américains, qui viennent la nuit bombarder les usines Krupp, à Essen. [...]

Impossible pour cette très jeune observatrice solitaire de ne pas ressentir, au-delà des discours, des paroles enflammées par l'alcool, la peur et la douleur qui sourdent des corps, les gestes, les tremblements qui échappent. Elle ne comprend rien. Mais ses yeux ont tout vu, son corps a tout enregistré de ces langages d'autant plus poignants qu'ils sont muets ${ }^{5}$. (párr. 3 y 4 )

Algo tan doloroso, tan demoledor como la guerra, tan impregnado de miedo, de ira, de terror, que ensordece las narraciones. El cuerpo enmudece, las palabras no fluyen. Aturdido, tembloroso, sobresaltado, intimidado, incapaz de articular sonidos, pero con las impresiones grabadas en la piel, en las vísceras. En lo superficial y en lo más profundo, en lo emocional, a través de sensaciones que traspasan, que se filtran, que se inscriben a un nivel más hondo e inconsciente. El psiquiatra Bessel van der Kolk (2015) nos habla de cómo tras episodios traumáticos el cuerpo también da buena cuenta de la experiencia, y el recuerdo del trauma queda codificado no solo en la mente sino también en las vísceras y en nuestra capacidad sensitiva, afectando a nuestra interacción emocional con el contexto. "El trauma es una huella dejada por una experiencia en la mente, el cerebro y el cuerpo. Esta huella tiene consecuencias permanentes sobre el modo en que el organismo humano logra sobrevivir en el presente" (p. 23). El trauma cambia nuestra mente, reconfigura nuestro cerebro, reorganiza nuestra percepción, pero también remodela nuestro cuerpo, en su fisionomía y su fisiología. Por eso, para la superación del trauma no es suficiente con enseñar a la mente a cambiar su modo de pensar y de percibir la realidad, también es necesario que el cuerpo recupere su sensibilidad y aprenda a conectarse de nuevo con las experiencias presentes. Porque las experiencias más devastadoras quedan inscritas también en el cuerpo, a través de sensaciones terribles puramente físicas. Un dolor físico que se hace insoportable y que escapa a nuestra razón. “...la solución requiere encontrar maneras de ayudar a la gente a modificar el paisaje sensorial interior de su cuerpo" (Van der Kolk, 2015, p. 83).

\footnotetext{
La niña aún no tiene cinco años y ya ha creado su lugar de juego entre las mesas. Quizá atrapa al vuelo las conversaciones, las palabras que aterrorizan. Las palabras de la guerra, del odio. Ella escucha probablemente a lo lejos los aviones de caza ingleses, más tarde americanos, que vienen a bombardear de noche las fábricas Krupp en Essen. [...]

Imposible para esa joven observadora solitaria no sentir, más allá del discurso, las palabras inflamadas por el alcohol, el miedo y el dolor que aturden que ensordecen los cuerpos, los gestos, los temblores que se escapan. No comprende nada. Pero sus ojos ya lo han visto todo, su cuerpo ha registrado todo de esos lenguajes tan conmovedores que resultan mudos. [trad. propia]
} 


\section{Cómo dar forma a los recuerdos de infancia}

"Ces souvenirs d'enfance sont vagues; je les ai oubliés. Ils reviennent pourtant dans mon travail. Je passe ma vie à essayer de donnerune forme à ces impressions évanouies, enfouies" ${ }^{\prime \prime}$ (Fretard, 1995, párr. 14). Y cuando los recuerdos afloran, lo hacen sobre el escenario. A través de su obra coreográfica, Pina Bausch se reencuentra con su infancia y rebusca en sus vivencias más tempranas.

The atmosphere of her early childhood seems to find an echo later in her pieces; music is heard, people come and go, and talk of their yearning for happiness. Yet her early experience of the war is also reflected in the pieces, in sudden outbursts of panic, fear of an unnamed danger ${ }^{7}$. (Servos, s.f., párr. 2)

Aquellas generaciones de artistas nacidos en las primeras décadas del siglo XX quedaron mudas tras la experiencia trágica de la guerra. Bausch nació con la guerra y la vivó de cerca en sus primeros años de vida. Pertenece a la primera generación de la posguerra, de la reconstrucción, y es capaz de enfrentarse al dolor descarnado de la guerra, y subir a escena la violencia, el terror, precisamente porque lo observó con toda su crueldad. Su cuerpo quedó conmovido por aquellas percepciones que no entendía pero sí podía sentir físicamente, registró las tragedias personales, los dramas de otros y otras, pero no el suyo propio, afortunadamente. Eso le permitió bucear en el dolor y en la incomprensión, caminar por las heridas del pasado, hablar a través de su cuerpo de aquel sufrimiento traumático, que era el de todos y todas, sin quedar paralizada por ello.

En sus coreografías se respira esa angustia vital, existencial, esa desesperanza que ella vivió pero que cualquier ser humano conoce de una manera u otra. "Hay un motivo último que impele a todos los bailarines: decir que no saben qué hacer con su tragedia personal e intentan colocarla, desesperada e inútilmente, en los demás." (Salas, 2015, p. 144). Sus creaciones están forjadas a partir de las aportaciones individuales de cada uno de los bailarines y las bailarinas. Composiciones colaborativas en las que cada persona asiste con su drama, sus deseos y sus miedos, sus incertidumbres y desasosiegos, una especie de desgarro común construido a partir de las tragedias personales. Una catarsis sentimental compartida.

Ella afirmaba que sus obras no eran autobiográficas, ni siquiera la emblemática Café Müller que tantas veces se ha vinculado a su infancia entre las mesas y sillas del restaurante que regentaban sus padres, "en cualquier caso sería autobiográfica de todos los que han trabajado en ella" (Salas, 2015, p. 132). Podríamos hablar entonces de su obra coreográfica como de una co-biografía construida colectivamente junto a sus bailarines y bailarinas, capaz de cartografiar las miserias y los temores más universales. Los visibiliza, los corporifica sobre el escenario, los muestra desnudos a través del movimiento, con toda honestidad y crueldad, porque el cuerpo nunca miente. $\mathrm{La}$ expresión de un cuerpo que baila no se puede disfrazar, no hay fraude posible.

Esos recuerdos de infancia son vagos; los he olvidado. Vienen sin embargo en mi trabajo. Me paso la vida intentando dar una forma a esas impresiones evanescentes, que huyen. [trad. propia]

La atmósfera de su primera infancia parece encontrar más tarde un eco en sus piezas; la música se oye, la gente viene y va y hablan de sus anhelos de felicidad. También su temprana experiencia con la guerra se refleja en las piezas, en repentinos arrebatos de pánico, miedo a un peligro sin nombre. [trad. propia] 
S'essayer, chercher en soi-même, trouver peut-être. Il est rare que l'auteur-chorégraphe-régisseur intervienne dans le déroulement de l'expérimentation et de l'auto-reconnaissance de son Groupe, - déroulement don't elle est l'insigatrice. [...] Très concentre, très calme, Pina Bausch suit la recherché de son groupe. $<<$ Chacun doit pouvoir être comme il le veut ou comme il s'est développé $>>[\ldots]<<\mathrm{Ce}$ que je fais: je regarde. Je n'ai jamais fait que regarder les gens. Je n'ai fait que voir, ou enssayer de voir les rapports humains, afin d'en parler. Voilà ce qui m'intéresse. Je ne connais d'ailleurs rien de plus important $>>^{8}$. (Hoghe, 1987, p. 8)

Pina Bausch no dejó nunca de ser aquella observadora silenciosa y tímida, discreta bajo una mesa. Incluso en el proceso creativo para elaborar sus coreografías parece que no hacía otra cosa más que observar a sus bailarines-coautores. Les provocaba mediante preguntas sorprendentes y extrañas, nunca imaginadas para conformar el origen de una danza, y esperaba sus respuestas verbalizadas, bailadas, interpretadas. Alain Platel, citado por Wiegand (2009), así lo describe: "She had little lists of questions. They could go from the absurd, like "What did you eat for Christmas?" to "How do you feel about love?" There were a thousand other questions in between. It was quite revolutionary"9 (párr. 1).

Después mira en silencio cómo improvisan, les deja tiempo para investigar, buscar el movimiento deseado, reflexionado, cada uno desde su personalidad y su manera de sentir, desde su experiencia y su comprensión del mundo. Cada cuerpo trabajando con su unicidad, cada personalidad aportando al conjunto. Un delicado camino introspectivo y personal, que se cruza con otros recorridos para desembocar en la obra colectiva y compartida. En los ensayos se mantiene callada, casi no habla $\mathrm{y}$, cuando lo hace, es en voz baja cerca de alguna persona. No da instrucciones generales (Hoghe, 1987, p. 9). No da indicaciones, ni dirige, no marca el camino a seguir, solo muestra el principio del sendero. Quizá por eso a esta coreógrafa le gustaba trabajar con los mismos bailarines, porque su proceso era lento. Algunos envejecen junto a ella, le acompañan durante décadas. No importa la edad, porque los años te hacen acumular experiencias y ese bagaje vital se hace fundamental para componer y enriquecer las piezas. Quiere bailarines y bailarinas con historias que contar, historias diversas, diferentes, únicas. Por eso busca danzantes de todos los continentes. "I pick my dancers as people. I don't pick them for nice bodies, for having the same height, or things like that. I look for the person, the personality"10 (Climenhaga, 2013, p. 88). Quiere bailarines que la inspiren y la ayuden a pensar, a interrogarse, a emocionarse. Bausch observa, trabaja con la emoción y con el cuerpo, desde cada emoción y cada cuerpo, desde muchas emociones y muchos cuerpos. Tal y como de niña hacía bajo las mesas del restaurante familiar.

Ensayar, buscar en uno mismo, encontrar quizá. Es raro que el autor-coreógrafo-regidor intervenga en el desarrollo de la experimentación y del auto-reconocimiento de su grupo, - desarrollo del que ella es la instigadora. [...] Muy concentrada, muy tranquila, Pina Bausch sigue la investigación de su grupo. $<<$ Cada uno debe poder ser cómo quiera o cómo se ha desarrollado $>>[\ldots]<<$ Lo que yo hago es mirar. No he hecho nunca nada más que mirar a la gente. No he hecho más que ver, o intentar ver las relaciones humanas con el objetivo de hablar de ellas. He aquí lo que me interesa. No conozco por otra parte nada más interesante $>>$. [trad. propia]

9 Tenía pequeñas listas de preguntas. Podrían ir desde el absurdo, como “¿Qué comiste para Navidad?” a “Cómo te sientes sobre el amor?" Había otras mil preguntas. Fue bastante revolucionario. [trad. propia]

10 Escojo a mis bailarines como personas. No los elijo por sus atractivos cuerpos, por tener la misma altura, o cosas así. Busco a la persona, la personalidad. [trad. propia] 


\section{El cuerpo como transmisor de la memoria colectiva del ser humano}

"Dès 1974, elle catapulte auprès d'un public incrédule et furieux une série de chorégraphies dédiées à tutes les victimes. Qu'elles soient sacrifiées à l'autorité d'un père, à la raison d'Etat, au manque d'amour, à la folie meurtrière"11 (Fretard, 1995, párr. 8). Podríamos afirmar que la obra de Pina Bausch está hablando, a través de cada una de las tragedias individuales y únicas que muestra en escena, de traumas que no dejan de ser colectivos. Es la universalización del drama humano, de los avatares del alma humana. Ese drama universal que Pina sube al escenario, no es más que el recuerdo, el reencuentro de cada uno con las experiencias de su pasado, de su infancia. Incluso aquellas que no se filtraron a través de la razón; aquellas que se inscribieron y quedaron codificadas corporalmente. Temor, espanto, pavor, pánico, desasosiego, desazón, el miedo somatizado. Catarsis en movimiento, catarsis que se origina desde el pensamiento y desde el sentimiento. Pensar a través del movimiento, sentir, reflexionar, dejar al cuerpo encontrar su gesto, personal e inconfundible, desde lo más profundo de su emoción.

La raíz etimológica de la palabra emoción, del latín emotio, movimiento, impulso, aquello que te mueve hacia, que proviene del verbo emovere, moverse, nos da la primera pista. Las emociones son impulsos, aquello que nos mueven hacia algún sitio, que nos motivan a la acción. Emocionarse, conmoverse, moverse, actuar, reaccionar. Las emociones están en el origen de nuestra activación y motivación, son el motor imprescindible que nos conduce a la acción. Las emociones modelan nuestra realidad, nuestros pensamientos, nuestra mente, nuestro cuerpo, nuestras acciones y movimientos. Lo que nos conmueve por dentro es el origen de todo lo demás. Edmund Gleede, citado por Adolphe (2007), escribe en el programa de mano de Barbe-Bleue, en 1977: "L'intérêt ne porte pas sur la torture et le meurtre, mais sur les pensées, les sentiments, les pulsions et les instincts qui conduisent à ces extrémités: ce qui a de l'importance pour Pina Bausch, ce sont les motivations"12 (p. 13). Bausch se interesa por aquello que conmueve al ser humano, por el origen de las pasiones y los horrores de su alma. Pero más allá de causas psicológicas, su interés se centra en cómo esos impulsos universales se transforman en movimiento según cada individuo. "Not "Why do you cry?" with all of its attendant psychological motivation, but the more elusive "How?" She is looking for the way in which each individual contains his or her expression, how it lives in his or her body"13 (Climenhaga, 2013, p. 59). Cada persona, cada personalidad da forma y corporalidad a sus sentimientos de una manera distinta, se expresa a través del movimiento de forma diferente, según su modo de habitar el cuerpo, de conquistar el espacio.

Al fin y al cabo, vivir, es un constante habitar de espacios. Apropiarse del aire, penetrar en él y dejar que él penetre en nosotros, sentirlo a nuestro alrededor, expandirlo, apoyarnos en él en busca de nuestro equilibrio, palparlo como parte de nuestra

11 Desde 1974, ella catapulta hacia un público incrédulo y furioso una serie de coreografías dedicadas a todas las víctimas. Ya sean las sacrificadas por la autoridad de un padre, por la razón de Estado, por la falta de amor, por la locura homicida. [trad. propia]

12 El interés no está en la tortura y el asesinato sino en los pensamientos, los sentimientos, las pulsiones y los instintos que conducen a estos extremos: esto es lo importante para Pina Bausch, esto son las motivaciones. [trad. propia]

13 No “¿Por qué lloras?” Con toda la carga psicológica que acompaña, sino la más elusiva “¿Cómo?”. Está buscando la manera en que cada individuo contiene su expresión, cómo vive en su cuerpo [trad. propia] 
vida,... todas estas son experiencias cotidianas en nuestra existencia; también en la danza. Podríamos decir que de una manera más profunda en la danza. La danza nos permite todo esto, experimentar con nuestro cuerpo, con nosotros mismos, conocernos un poco mejor, situarnos en el mundo, reinventarnos, significarnos y vincularnos con nuestros semejantes. Nos conectamos al mundo y lo interpretamos a través del cuerpo. De esta forma nuestro cuerpo se transforma en un terreno privilegiado para la reflexión y la representación de nuestra propia identidad, individual y social. Nuestros gestos nos significan y diferencian frente a los demás, pero al mismo tiempo nos hermanan y nos identifican a ellos y ellas.

These parades of human behaviour are often found in Bausch's pieces, tired and aggressive, embarrassed and arrogant, anxious and searching, tense and friendly, gestures are the visible reactions to invisible situations. The seemingly personal and yet universal gestures, not placed in a (theatre) story become the story itself, and reflect the traces of a lived and unlived life. "We really are quite transparent if we just look at each other. The way someone walks or how they carry themselves says something about the way they live, or about what happened to them" ${ }^{14}$. (Climenhaga, 2013, p. 73)

Sobre el escenario situaciones de la vida real, experiencias vitales que son personales e íntimas pero también comunes y compartidas. Bausch es capaz de llegar al gesto universal, a los gestos que antropológicamente nos pertenecen como especie gracias a la evolución humana. Se trata de redescubrir, de volver a encontrar aquello que nos vincula desde nuestros ancestros, pero no de manera razonada, sino corporeizada. "Le processus n'est pas mental, mais organique"15 (Fretard, 1995, párr. 13), afirma la coreógrafa. Cada uno desde su unicidad corporal. Cada cuerpo como fuente inagotable de gestos, de movimientos, de ideas danzadas. Ese es el verdadero descubrimiento en Bausch. El cuerpo como transmisor de la memoria colectiva del ser humano, interpretada desde cada individualidad.

La obra coreográfica de Pina Bausch nos muestra un cabaret de pasiones (Adolphe, 2007, p. 11) capaz de exponer la humanidad desnuda del movimiento. Una cosa determina la otra y ella quería llegar al origen, a la emoción, a la pasión que nos conmueve, para encontrar la expresión, el movimiento, el gesto más verídico y puro, ese gesto universal. La verdad y la autenticidad como camino hacia la belleza.

\section{Catarsis acompañada, acompasada, compartida}

El espectador se convierte entonces en ese observador discreto y silencioso, como si Bausch le cediese durante la representación aquel lugar escondido bajo las mesas

\footnotetext{
14 Estos desfiles de la conducta humana se encuentran a menudo en las piezas de Bausch, cansados y agresivos, avergonzados y arrogantes, ansiosos y buscando, tensos y amistosos, los gestos son las reacciones visibles a situaciones invisibles. Los gestos aparentemente personales y a la vez universales, no colocados en una historia (de teatro) se convierten en la historia misma, y reflejan las huellas de una vida vivida y no vivida. "Somos muy transparentes si nos miramos unos a otros. La forma en que alguien camina o cómo se llevan a sí mismos dice algo sobre la forma en que viven, o sobre lo que les sucedió." [trad. propia]

15 El proceso no es mental, es orgánico. [trad. propia]
} 
del restaurante familiar. El escenario como una ventana abierta por la que observar la vida pasar, la aleatoriedad de la vida, lo azaroso de nuestras existencias bajo los focos de la escena. Vemos no solo tragedias tan aterradoras como la guerra que ella vivió, también otras tragedias cotidianas, miserias contemporáneas. La opresión, la humillación de los débiles, el abandono, el rechazo, la angustia, la frustración, la conflictividad y complejidad de las relaciones humanas, la incomunicación con la pareja, la incomunicación social. Porque las experiencias traumáticas nos incomunican, nos desconectan de la realidad, de nuestro contexto y de nosotros mismos, de nuestros cuerpos. Cuerpos disociados física y socialmente, desincronizados con sus semejantes.

La cuestión crítica es la reciprocidad: ser realmente escuchado y visto por las personas que nos rodean, sentir que tenemos el apoyo de alguien en su mente y en su corazón. Para que nuestra fisiología se calme, se cure y crezca necesitamos una sensación visceral de seguridad. (Van der Kolk, 2015, p. 87)

Conectar con los otros, las otras, sentirse seguro a su lado, ser capaz de establecer relaciones sociales e interpersonales seguras y satisfactorias es la base de nuestro equilibrio interior, mental y corporal. Y consecuentemente, este apoyo social es un elemento fundamental en la recuperación de personas anuladas por un trauma. Actividades que nos permiten sentirnos conectados con aquellos y aquellas que nos rodean, que requieren de una experiencia compartida de sincronía, cooperación y entendimiento común son placenteras y gratificantes; nos hacen sentirnos vivos y tener una existencia plena. Compartir nuestro ritmo vital y acompasarlo con el de los demás es fundamental. Algunas terapias no farmacológicas para el tratamiento del trastorno por estrés postraumático apoyan tratamientos que incorporan técnicas corporales de relajación y conciencia corporal, de estimulación sensorial, o de juego e interacción social. De esta manera, trabajan con dos objetivos: calmar las tensiones físicas y restablecer relaciones sociales seguras. Y entre estos tratamientos se incluyen actividades como el canto y la danza comunitaria (Van der Kolk, 2015, p. 95).

Un grupo de adultos de un centro de día de la Red Pública de Salud Mental marcan un ritmo con los pies mientras configuran una danza circular y comentan que este gesto les recuerda la sensación de pisar hormigas. Así pueden expresar agresión, enojo o alguna emoción similar de un modo que no les produce angustia, además de desplegar un pensamiento simbólico. (Wengrower y Chaiklin, 2008, p. 45-46)

Las coreografías de Pina Bausch tiene algo de esto; la desesperanza, el miedo, la angustia, el drama individual, que se transforma en universal y simbólico sobre el escenario, para ser compartido en una especie de catarsis acompañada, acompasada, de la que sus bailarines y bailarinas no salen indemnes. Cada uno custodiado por sus fantasmas, aislado, buscando la comunicación con el prójimo, que no siempre llega, y utilizando el movimiento como elemento catalizador, capaz de remover sentimientos y crear nuevos vínculos. Buscando a través de sus experiencias pasadas, de sus vivencias corporales, la conexión presente con el mundo, el apoyo en los otros, las otras, para calmar el sufrimiento y curar el trauma. En este sentido, el proceso de introspección sensorio-emocional a través del movimiento compartido que desarro- 
lla Bausch se asemeja a las terapias artístico-creativas relacionadas con lo corporal que trabajan desde el potencial terapéutico de los procesos creativos a través de la danza y el movimiento. Tomando la expresión de Van der Kolk (2015) podríamos decir que, de alguna manera, Bausch está investigando sobre cómo el paisaje sensorial interior del cuerpo se construye y cómo puede ser transformado y regenerado cuando nos movemos juntos, sincronizando nuestros ritmos y adaptándonos unos a otros (83).

La actividad artística puede ser una poderosa vía de expresión y comunicación de nuestras emociones no conscientes, de nuestras ansiedades y angustias soterradas, capaz de proporcionarnos beneficios terapéuticos para nuestro bienestar físico, psicológico y nuestro desarrollo personal y social. “...sentimientos que de otra forma serían penosos de soportar pueden ser tolerados, sostenidos, gracias a su presentación por la vía artística-simbólica" (Wengrower y Chaiklin, 2008, p. 45). Las terapias artísticas buscan el mostrar para no decir, especialmente cuando la palabra no es suficiente para curar, y nos proponen procesos creativos que indagan en el conocimiento interior a través de nuestros sentimientos y pensamientos, vinculando de esta manera cuerpo, emoción y cognición (López y Martínez, 2006, p.13).

Atenta, el arteterapia señala momentos de reflexión, anida, en un espacio suspendido, las emociones que el sujeto hace aflorar, acompaña al que crea y ayuda a que la reflexión de lo visible y de lo palpable surja, así como también de lo invisible y de lo impalpable. Despliega un tiempo creador y reflexivo, un tiempo estético donde se funden las emociones, las experiencias, la necesidad de saber de uno y la necesidad de saber del mundo. [...] Ocurre en un espacio y un tiempo fuera del espacio y el tiempo, pero paradójicamente ligado a ambos, ligado a la vida. [...]... busca en el proceso creador una vía tercera, terciaria, de seguir imbricando el conocimiento de sí y del otro con la emoción de saberse vivo a través de sus sentidos. (López y Martínez, 2006, p.16)

Baush también acompaña a sus bailarines cuando crean, cuando exploran, y ellos la acompañan a ella, en sus reflexiones, en su necesidad por indagar en las emociones humanas, de bucear en el análisis de las relaciones interpersonales, para entenderse, entendernos, un poco mejor. También sus coreografías presentan ese espacio suspendido, ese tiempo creador y reflexivo, construido a partir de emociones y experiencias reales. Para a través del cuerpo hacer visible lo invisible y lo impalpable de nuestras vidas, conectando de forma inmediata lo que ocurre en la sala de ensayos, o en el escenario, con nuestra cotidianeidad. "Watching Bausch's work, you become aware of your own perspective on people and relationships, you try out alternative views and are forced to acknowledge that the world is bigger than your own experience"16 (Climenhaga, 2013, p. 65). Por ello Bausch es capaz de universalizar el trauma, porque lo que allí sucede nos vincula a todos y todas. No solo a los que actúan sobre la escena, también a los espectadores y las espectadoras que observan y se descubren en ellos y ellas. Una suerte de redención, quizás no liberación, sino más bien un acto de constricción colectivo (Salas, 2015, p. 145).

16 Observando el trabajo de Bausch, tomas conciencia de tu perspectiva personal sobre las personas y las relaciones humanas, pruebas puntos de vista alternativos y te ves obligado a reconocer que el mundo es más grande que tu propia experiencia. [trad. propia] 
Bausch confiesa: "Sometimes, we can only clarify something by confronting ourselves with what we don't know. And sometimes the questions we have bring us back to experiences which are much older" ${ }^{17}$ (Popp, s.f., párr. 2). Interrogantes abiertos que nos cuestionan, hacen que nos preguntemos sobre nosotros mismos, nuestro pasado y presente, nuestra posición ante la realidad que vivimos. Nos colocan ante nuestros deseos rotos, temores y dramas, ante todas esas experiencias que nos desequilibran a diario, ante nuestras derrotas personales, dificultades cotidianas para mantenernos seguros en nuestro cuerpo, en nuestro mundo. "Pina Bausch theatre admits doubts and insecurities, and includes corners and edges that you can bump against to rip open old wounds and release repressed feelings. [...] Her pieces ask questions. Answers remain open."18 (Climenhaga, 2013, p. 66). No hay respuestas. En ese sentido no falta una perspectiva derrotista ante el conflicto. Y cruel. Salas (2015) en un obituario sobre la coreógrafa, publicado en el diario El País, hablaba así sobre Claveles, pieza coreografiada por Bausch en 1982:

Era un patético canto a lo efímero, otro elemento vital de su estado. Chispazos de humor corrosivo, autohumillaciones explícitas, Bausch rebuscaba con cierta crueldad en las almas de sus artistas, las vaciaba y las volvía a poblar de una angustia que ya era otra, coloreada por su toque abrumador. (p. 139)

Bausch nos vapulea, desordena nuestro pensamiento, nos saca de nuestro bienestar ordenado y bienpensante, y todo queda ahí. Muestra a través de la expresión danzada sentimientos que no puede clasificar, ni definir, solo transcribir (Hoghe, 1987, p. 21). Sin consuelo, ni solución, aunque quizá con la esperanza de que el camino está en la reflexión compartida entre todos, viendo nuestro reflejo en los otros, en las otras, subido a un escenario, legitimado, dignificado, lleno de verdad y por tanto de belleza.

\section{Referencias bibliográficas:}

Adolphe, J. M. (2007). Corpus Pina Bausch. En G. Delahaye, Pina Bausch (pp. 9-23). Arles: Actes Sud.

Bausch, P. (2007). What moves me, [en línea]. Wuppertal: Pina Bausch Foundation. Disponible en: http://www.pinabausch.org/en/pina/ what-moves-me [2017, 11 de julio]

Climenhaga, Royd (Editor) (2013). The Pina Bausch sourcebook. The making of tanztheater. New York: Routledge.

Fretard, D. (1995, 10 de febrero). Pina Bausch, l'exorciste. Le Monde.fr Archives [en línea]. Disponible en: http://www.lemonde.fr/acces- restreint/archives/article/1995/02/10/afb0bda5be54b621ce77d928a8ebabfe_3834939_1819218.html [2017, 6 de agosto]

Hoghe, R. (1987). Pina Bausch. Histoires de Théâtre dansé. París: L'Arche.

López Fernández Cao, M. y Martínez Díez, N. (2006). Arteterapia. Conocimiento interior a través de la expresión artística. Madrid: Ediciones Tutor.

Popp, U. (Sin fecha). Season 2017-2018, (Trad. J. Callejen, S. Jacobs y S. Morris). [en

17 A veces, sólo podemos aclarar algo al confrontarnos con lo que no sabemos. Y a veces las preguntas que tenemos nos devuelven a experiencias que son mucho más antiguas. [trad. propia]

18 El teatro de Pina Bausch admite dudas e inseguridades, e incluye esquinas y bordes con los que puedes chocar para rasgar viejas heridas abiertas y liberar los sentimientos reprimidos. [...] Sus piezas hacen preguntas. Las respuestas permanecen abiertas. [trad. propia] 
línea]. Wuppertal: The Tanztheater Wuppertal Pina Bausch. Disponible en: http:/www. pina-bausch.de/en/index.html [2017, 11 de julio]

Salas, R. (2015). ¿Por qué bailamos? Papelería sobre la danza (y el ballet) Volumen III. Barcelona: Ediciones Cumbres.

Servos, N. (Sin fecha). Talking about People through Dance - Pina Bausch Biography, (Trad. S. Morris) [en línea]. Wuppertal: Pina Bausch Foundation. Disponible en: http:// www.pinabausch.org/en/pina/biography [2017, 11 de julio]

Van der Kolk, B. (2015). El cuerpo lleva la cuenta: cerebro, mente y cuerpo en la superación del trauma. Barcelona: Editorial Eleftheria.

Wengrower, H. y Chaiklin, S. (coords.) (2008). La vida es danza. El arte y la ciencia de la Danza Movimiento Terapia. Barcelona: Gedisa Editorial.

Wiegand, Ch. (2009, 3 de Julio). Pina Bausch tributes: 'She got the keys to your soul'. The Guardian. [en línea]. Disponible en: https://www.theguardian.com/stage/2009/jul/03/ pina-bausch-tributes [2017, 20 de julio] 